Voix et Images

voixetimages

\title{
Bibliographie de Monique Bosco
}

\section{Myrianne Pavlovic}

Volume 9, numéro 3, printemps 1984

Monique Bosco

URI : https://id.erudit.org/iderudit/200480ar

DOI : https://doi.org/10.7202/200480ar

Aller au sommaire du numéro

Éditeur(s)

Université du Québec à Montréal

ISSN

0318-9201 (imprimé)

1705-933X (numérique)

Découvrir la revue

Citer ce document

Pavlovic, M. (1984). Bibliographie de Monique Bosco. Voix et Images, 9(3),

55-82. https://doi.org/10.7202/200480ar d'utilisation que vous pouvez consulter en ligne.

https://apropos.erudit.org/fr/usagers/politique-dutilisation/ 


\title{
Bibliographie de Monique Bosco
}

\author{
par Myrianne Pavlovic, Université de Montréal
}

Les écrits de Monique Bosco, de même que les commentaires qu'ils ont suscités, n'avaient fait l'objet d'aucune recension jusqu'à présent. Éparses, incomplètes, souvent inexactes, les références bibliographiques disponibles devaient sans cesse être vérifiées.

L'inventaire proposé ici, à défaut d'être exhaustif, est juste, sauf peutêtre sous la rubrique "textes radiophoniques», où subsistent quelques imprécisions. Par contre j'ai accordé une attention particulière aux textes de Monique Bosco publiés dans les revues et les journaux, en dépouillant systématiquement, semaine après semaine ou mois après mois, les numéros du Devoir, de la Presse et du MacLean. La deuxième partie de la bibliographie (études et critiques) gagnera certainement à être mise à jour; j'ai consulté les principaux journaux et les principales revues au moment des publications de M. Bosco, j'ai vérifié les références déjà existantes mais le repérage, ici, n'a rien de systématique. On notera enfin que toutes les rubriques sont présentées selon un ordre chronologique croissant, des textes les plus anciens aux plus récents.

On recevra donc cette bibliographie comme un travail préliminaire que des recherches futures veilleront à compléter.

La bibliographie se présente comme suit:

1. Ecrits de Monique Bosco
A - Romans, récits
B - Nouvelles et textes
C - Poèmes
D - Texte dramatique
E - Textes radiophoniques 
F - Communications, débats

G - Articles dans les revues et journaux 1 - Dans MacLean

2 - Dans la Presse

a) Israël

b) USA

3 - Dans le Devoir

a) télé et radio

b) télé

4 - Articles divers

$\mathrm{H}$ - Entrevues

11 - Études et critiques
A - Sur les romans et récits
B - Sur les poèmes
C - Sur les textes radiophoniques
D - Sur les articles
E - Sur l'ensemble de l'oeuvre

\section{LES. ÉCRITS DE MONIQUE BOSCO}

\section{A - ROMANS, RÉCITS}

- Un amour maladroit, Paris, Gallimard, 1961, 213 p.

- Les Infusoires, Montréal, HMH, «L'Arbre» no 7, 1965, 174 p.

- La Femme de Loth, Paris/Montréal, Laffont/HMH, 1970, 282 p.

- Lot's Wife, Toronto, McClelland and Stewart, 1975, 149 p. (trad. John Glassco)

- New Medea, Montréal, L'Actuelle (Quinze), 1974, 149 p.

- Charles Lévy, m.d., Montréal, Quinze, 1977, 136 p.

- Portrait de Zeus peint par Minerve, Montréal, HMH, 1982, 179 p.

\section{B - NOUVELLES ET TEXTES}

- «Le menteur d'Amsterdam», Châtelaine, Montréal, 2:6, juin 1961, p. 30 et 52-56.

- «Mes amours», Châtelaine, Montréal, 3:2, février 1962, p. 38 et 52-56.

- «Remous», Liberté, Montréal, 4:22, avril 1962, p. 211-218.

- «Le chapeau rouge d'Antoinette», Châtelaine, Montréal, 7:10, octobre 
1966, p. 36, 37, 93, 94, 95, 96, 98.

- «Corps-mort», la Barre du jour, Montréal, nos 56-57, mai-août 1977, p. 68-83.

- «Mooring-Buoy/Moored Body», Room of One's Own, Vancouver, 4:1-2, 1978, p. 44-45 (trad. Josée M. Leblond).

- «Du sang à la six», la Nouvelle Barre du jour, Montréal, nos 68-69, septembre 1978, p. 45-50.

- «Mauvais rêve», Possibles, Montréal, 4:1, automne 1979, p. 67-71.

- «Alexandria», la Nouvelle Barre du jour, Montréal, no 132, novembre 1983, p. 67-75.

\section{C - POÈMES}

- «Poèmes», Écrits du Canada français, Montréal, Hurtubise, no 15, 1963, p. 167-191.

- «Poèmes», Europe, Paris, 47:478-479, février-mars 1969, p. 184-190.

- Jéricho, Montréal, HMH, «Sur Parole», 1971, 63 p.

- Schabbat 70-77, Montréal, Quinze, 1978, 98 p.

\section{D - TEXTE DRAMATIQUE}

- «Le cri de la folle enfouie dans l'asile de la mort», Écrits du Canada français, Montréal, Hurtubise, no 43, 1981, p. 29-75.

\section{E - TEXTES RADIOPHONIQUES}

- «Zola», présenté à Radio-Canada bande MF, dans le cadre de la série Horizons, diffusée le 18 avril 1976 (M. Bosco parle de ce que la lecture de Zola représente pour elle. Réalisation Gilles Archambault).

- «Venise», présenté à Radio-Canada bande MF, en septembre 1976 (M. Bosco parle de Venise avec l'animateur Normand Biron).

- "Le cri de la folle enfouie dans l'asile de la mort», présenté à RadioCanada bande MF, dans le cadre de la série Premières, le 22 septembre 1978 (réalisation Madeleine Gérôme , musique Gabriel Charpentier, voix de Dyne Mousso. Soumis pour le Prix Italia et présenté également à Radio-Zagreb en décembre 1978).

- «Poésie», présenté à Radio-Canada bande MF, en 1981 (?) (Réalisation Raymond Fafard).

- "Journal intime», présenté à Radio-Canada bande MF durant la semaine du 2 août 1982 (Réalisation André Major, voix de Gaétane Laniel, cinq émissions). 


\section{F - COMMUNICATIONS, DÉBATS}

- «Télévision et cinéma sont-ils frères ennemis? Un entretien à quatre préparé par Jean Basile», le Devoir, 53:141, 16 juin 1962, p. 11 et 10.

- «La part de l'enseignement de la littérature dans l'acquisition d'une culture littéraire», Liberté, Montréal, 10:3 (no 57), mai-juin 1968, p. 52-57 (Actes de la VIe Rencontre des écrivains sur les Écrivains et l'Enseignement de la littérature).

On pourra lire aussi sur cette Rencontre:

- BOSCO, Monique «Libre opinion-Dissidence ou l'unanimité renaît de ses cendres», le Devoir, Montréal, $59: 131,4$ juin 1968, p. 4 et 5 .

- BOSCO, Monique "Contre l'unilinguisme au Québec», la Presse, Montréal, 84:131, 5 juin 1968, p.4.

- GODIN, Gérald «Deux réponses à Monique Bosco: ce qui s'est passé à la VIe Rencontre des Écrivains», le Devoir, Montréal, 54:136, 10 juin 1968, p. 4.

- GODIN, Gérald "Gérald Godin répond à Monique Bosco», la Presse, Montréal, 84:135, 10 juin 1968, p. 4.

- BERTRAND, Paul «Nationalisme vs démocratie», la Presse, Montréal, 84:152, 2 juillet 1968, p. 6 (réponse à $G$. Godin).

- JASMIN, Claude «La peur du nationalisme aveugle Monique Bosco», le Devoir, Montréal, 59:137, 11 juin 1968 , p. 4.

- PAYETTE, André «Explications à la suite d'une rencontre d'écrivains", la Presse, Montréal, 84:140, 15 juin 1968, p. 4.

- «La femme et l'écriture», Liberté, Montréal, 18:4-5 (nos 106-107), juillet-octobre 1976, p. 74-81 (Actes de la Rencontre des écrivains sur la Femme et l'écriture).

- «Les filles de Virginia Woolf», Colloque du Collège Rosemont, Écriture et Société, animé par Louise Bouchard, le 24 avril 1980 (un vidéo est disponible).

\section{G - ARTICLES DANS LES REVUES ET JOURNAUX}

1- Dans le Magazine MacLean (chronique littéraire mensuelle, sauf pour les titres marqués d'un *).

- «Les apprentis-sorciers» $2: 4$, avril 1962, p. $26,27,28,63$.

- «Dans le désert de nos oeuvres littéraires une oasis: Jean Le Moyne» 
3:1, janvier 1963 , p. 48-49.

(sur Convergences de Le Moyne et Un homme en laisse de JeanPaul Filion)

- «De Maria Chapdelaine à ... Tristan, les bleuets sucrés» 3:2, février 1963, p. 46-47.

(sur les Enfants qui s'aiment et Autour de toi Tristan, de Claire France)

- «Une littérature de 'quai de gare' qui a manqué le train» 3:3, mars 1963, p. 62. (sur Prix David de Charles Hamel)

- "Les Montréalais: un scénario qui manque de chair» 3:4, avril 1963, p. 68-69. (sur ce recueil de nouvelles d'Andrée Maillet)

- «Laberge, un grand nouvelliste qui sort de l'ombre après 60 ans» 3:5, mai 1963, p. 79. (sur Anthologie d'Albert Laberge)

- "Poupée: une longue promenade triste devant le miroir» 3:6, juin 1963, p. 82-83. (sur ce roman de Claire Mondat)

- "Thériault renonce à l'exotisme de ses derniers romans» 3:8, aout 1963, p. 50.

(sur le Grand Roman d'un petit homme)

- «L'inspecteur Tanguay de Gagnon, c'est Maigret sans Simenon» 3:9, septembre 1963, p. 70.

(sur Meurtre sous la pliuie de Maurice Gagnon et Quatre Montréalais en l'an 3000 de Suzanne Martel)

- "Le Charivari, best-seller aux U.S.A., n'émeut pas au Québec» 3:10, octobre 1963, p. 86.

(sur ce roman de Robert Goulet)

- "L'or des Indes, de Gélinas: le délire d'être sans passé» 3:11, novembre 1963, p. 91. (sur ce roman de Pierre Gélinas)

- "Amadou de Louise Forcier, un roman qui me scandalise» 3:12, décembre 1963, p. 91. (sur ce roman de Louise Maheux-Forcier)

- «Depuis peu le roman connaît la sexualité» 4:2, février 1964, p. 46.

(sur Inutile et Adorable de Roger Fournier)

- "Simone en déroute: pas de 'message'» 4:3, mars 1964, p. 63.

(sur ce roman de Claude Mathieu)

- «Festival du Gesu: Sartre est interdit» 4:4, avril 1964, p. 70-71.

(sur la situation du théâtre au Québec) 
- «Document: le roman d'une libération» 4:5, mai 1964, p. 79.

(sur la Ville inhumaine de Laurent Girouard)

- «Jasmin, Benoît, découvertes du Salon du Livre» 4:6, juin 1964, p. 79.

(sur Ethel et le terroriste de Claude Jasmin et Quelqu'un pour m'écouter de Réal Benoît)

* - «Arrêtée à New-York dans une manifestation antiraciste, l'écrivain Monique Bosco livre ses réflexions sur le problème noir» 4:7, juillet 1964, p. 53-54.

* - «Le racisme au jour le jour» $4: 10$, octobre 1964 , p. $38,39,76,77,78,79,83$. (dans les États du Sud, la Louisiane en particulier)

- "Téléthéâtre: une année de vaches maigres» 4:10, octobre 1964, p. 93.

- «Le temps des cerises et celui du cocotier» 4:11, novembre 1964, p. 101-102.

(sur la situation difficile de la jeunesse, en littérature comme ailleurs)

* «À mesure que les 'autres', les Anglais, s'améliorent, nous devenons plus 'plates'" $5: 1$, janvier 1965 , p. 26 et 43. (dans la chronique "Sauf vot' respect")

- «Pourquoi pas une 'machine' à long métrage?» 5:1, janvier 1965, p. 46. (sur la Machine à écrire de Jean-A. Beaudot)

- «Trop, beaucoup trop de livres!» 5:2, février 1965, p. 47. (entre autres le Poisson pêché de Georges Cartier, le Cassé de Jacques Renaud, le Cabochon d'André Major, le Sortilège de Marcel Proust de Marcel Valois et l'Écrivain et son Theátre de Paul Toupin)

- "Quand les critiques sautent la clôture» 5:3, mars 1965, p. 60. (sur Nouvelles singulières de Jean Hamelin, la Jument des Mongols de Jean Basile, Dis-mois que je vis de Michèle Mailhot et Survivre d'Alice Parizeau)

- «Enfin, un vrai roman» 5:4, avril 1965 , p. 58. (sur les Remparts de Québec d'Andrée Maillet)

- «Et si Hébert était jugé par ses pairs?»

5:5, mai 1965 , p. 93.

(sur la Vie à Trois de Gilles Archambault, l'Eau est profonde de Diane Giguère, Pour une conversion de la pensée chrétienne de Fernand Dumont, le Temps et l'Espace dans l'oeuvre de Paul Claudel, de G.-André Vachon, et sur l'affaire Jacques Hébert) 
- «Un roman, deux prix littéraires»

$5: 6$, juin 1965, p. 63.

(sur les Terres sèches de Jean-Paul Pinsonneault, Ethel et le terroriste de Claude Jasmin et le Couteau sur la table de Jacques Godbout)

- «Le nouveau Bessette: renouveau»

5:7, juillet 1965, p. 46.

(sur l'Incubation)

- «Pas de vacances pour les critiques!»

5:9, septembre 1965 , p. 60-61.

(sur le Funambule de Wilfrid Lemoine, Retour a Coolbrook de Gilles Marcotte, Pleure pas Germaine de Claude Jasmin et les Terres noires de Jean-Paul Fugère)

- «Enfin un style Marie-Claire Blais» 5:10, octobre 1965, p. 70-71.

(sur Une Saison dans la vie d'Emmanuel)

- «Un m... bon roman»

5:11, novembre 1965 , p. 92-93.

(sur Ce maudit Soleil de Marcel Godin, la Dormeuse éveillée d'Yvette Naubert et la Fleur de peau d'Hélène Ouvrard)

- «Un grand cahier du Cercle Juif» 5:12, décembre 1965, p. 67.

(sur les Juifs et la communauté française, premier Cahier du Cercle Juif)

- "Écrire est un grand amour»

6:1, janvier 1966, p. 45-46.

(sur Prochain Épisode d'Hubert Aquin)

- «Un titre bien choisi»

6:2, février 1966, p. 43.

(sur Une vie d'enfer d'André Laurendeau et Histoires galantes de Bertrand Vac)

- "Née d'un tigre et d'une colombe»

6:3, mars 1966, p. 46.

(sur Dans un gant de fer de Claire Martin)

- "Ceux qui n'ont pas eu le Prix...»

6:4, avril 1966, p. 70-71.

(sur la Chèvre d'or d'Anne Bernard, À nous deux de Roger

Fournier et le Passage de Minou Petrowski)

- «La peur du roman»

6:5, mai 1966, p. 73.

(sur la Mort exquise de Claude Mathieu, Nouvelles Montréalaises d'Andrée Maillet et Coeur de sucre de Madeleine Ferron)

- "Gabrielle Roy, fidèle à elle-même»

6:6, juin 1966, p. 66.

(sur la Route d'Altamont) 
- «Un public, de théâtre dont on se moque» 6:7, juillet 1966, p. 46-47.

(sur les créations théâtrales québécoises)

- "Au lieu de poursuivre un succès certain...» 6:8, août 1966, p. 43.

(sur l'Insoumise de Marie-Claire Blais)

- «... Mais planter à cet âge?»

6:9, septembre 1966, p. 58-59.

(sur le numéro 21 des Écrits du Canada français consacré aux Jeunes Auteurs et sur Contes pour buveurs attardés de Michel Tremblay)

- «Enfin, un film d'auteur» 6:10, octobre 1966, p. 75. (sur le film YUL 871 de Jacques Godbout)

- «Trois livres décevants»

6:11, novembre 1966, p. 78-79.

(sur Solange de Jean-Guy Pilon, la Joue droite de Claire Martin et le Monde sont drôles de Clémence Desrochers)

- «La plume d'Henry Bordeaux»

6:12, décembre 1966, p. 67.

(sur la Patience des justes de Pierre de Grandpré et l'Itinéraire de Simone Landry-Guillet)

- «Une chanson simple»

7:1, janvier 1967, p. 42.

(sur la Fugue d'André Berthiaume et le Temps du carcajou d'Yves Thériault)

- «Blais contre Ducharme»

$7: 2$, février 1967, p. 54.

(sur l'Avalée des avalés et Une Saison dans la vie d'Emmanuel)

- "Jadis, on appelait ça le démon du midi»

7:3, mars 1967 , p. 62.

(sur la Fin des loups-garous de Madeleine Ferron et Loreley de Yerri Kempf)

- «... lectures dont on se passerait»

$7: 4$, avril 1967 , p. 82 .

(sur Un homme, rue Beaubien de René Chicoine, le Soleil sur la façade d'Anne Bernard et les Rapides de Jean-F. Somcynsky)

- "Quatre pièces pour lire»

7:5, mai 1967, p. 82.

(sur le Marin d'Athènes de Réal Benoît, Encore cinq minutes et Un cri qui vient de loin de Françoise Loranger, et Joli Tambour de Jean Basile)

- «Un Ducharme génial»

7:6, juin 1967, p. 66-67.

(sur le Nez qui voque) 
- «La portion congrue à la chose écrite»

7:7, juillet 1967, p. 54-55.

(sur les romans lus à la radio de Radio-Canada le midi)

- «Quelques lectures d'été»

7:8, août 1967, p. 46-47.

(surtout sur Journal d'un Jeune Marié de Roger Fournier et le Portique de Michèle Mailhot)

- "On ne triche pas avec l'âge»

7:9, septembre 1967, p. 62-63.

(sur Un dos pour la pluie de Jean Hamelin)

- «Des lettres qui sont un vrai roman»

7:10, octobre 1967 , p. $92-93$.

(sur Lettres à ses amis de Saint-Denys Garneau)

- "Jean Basile fait son pesant d'or"

7:11, novembre 1967, p. 83.

(sur le Grand Khan)

- «Le mauvais exemple de nos cousins de France»

7:12, décembre 1967 , p. 78-79.

(sur Salut Galarneau de Jacques Godbout)

- «Un voyage poétique aux enfers»

8:1, janvier 1968, p. 46-47.

(sur David Sterne de Marie-Claire Blais)

- "Cancer: le hochet des prix littéraires»

8:2, février 1968 , p. 50 .

(sur ce roman d'Anne Bernard et sur Anna de Louis Gauthier)

- "Thériault fait du Thériault»

8:3, mars 1968 , p. 58.

(sur l'Appelante)

- «Le titre est long, la vertu courte»

8:4, avril 1968, p. 79.

(sur Je voulais te parler de Jeremiah, d'Ozelina et de tous les autres de Jean' O'Neil)

- «Tous frères sous le soleil»

8:5, mai 1968; p. 78-79.

(sur les Soleils des indépendances de Ahmadou Kourouma)

- «Ce 'cochon de payant' de lecteur»

8:6, juin 1968, p. 47.

(sur Trou de Mémoire de Hubert Aquin)

- «La mode du roman passe vite au ciné»

8:7, juillet 1968, p. 46.

(sur Kid Sentiment de Jacques Godbout et Un hiver trop doux d'Arthur Lamothe)

- "Les drames des aînés compris et incarnés»

8:8, août 1968, p. 46.

(sur la Guerre, Yes Sir de Roch Carrier et la Crise de la conscrip. tion d'André Laurendeau) 
- "Ả déguster en fin de saison" 8:9, septembre 1968, p. 58. (sur la Saison des artichauts de Réal Benoît et Aldébaran ou la Fleur de Claire de Lamirande)

- «Ethier-Blais au goût du jour» 8:10, octobre 1968, p. 74. (sur Mater Europa)

- «Tout ce qu'il faut pour la panse» 8:11, novembre 1968 , p. 78-79. (sur la ré-édition d'oeuvres littéraires québécoises)

- «Fraîcheur et pureté de Réjean Ducharme» 8:12, décembre 1968, p. 62-63. (sur l'Océantume)

- "Grâce à Blais et Ducharme, 1968 fut une grande année» 9:1, janvier 1969, p. 46. (sur Manuscrits de Pauline Archange et l'Océantume)

- "L' «écriture» en France ou ici, ça se ressemble» 9:2, février 1969, p. 55. (sur les coïncidences d'écriture chez les auteurs de la «francité»)

- «Étalons fabuleux et critiquables critiques» 9:3, mars 1969 , p. 54. (sur Kesten d'Yves Thériault, la Tutelle d'Alain Pontaut, le Vent du diable d'André Major, et les parutions récentes)

- «Enfin Ducharme vint...» 9:4, avril 1969, p. 90-91. (sur l'influence de Réjean Ducharme et sur Bien vôtre de Jean Nadeau)

- «Un menteur infiniment poétique» 9:5, mai 1969, p. 70. (sur Jimmy de Jacques Poulin)

- «Faut-il laisser mûrir les auteurs?» 9:6, juin 1969, p. 60-62. (sur Faire sa mort comme faire l'amour de Pierre Turgeon, Enfances brisées d'Émile Martel et Rimbaud, mon beau salaud! de Claude Jasmin)

2- Dans la Presse

a) Envoyée spéciale de la Presse, Monique Bosco fait un séjour de plusieurs semaines en Israël et publie à son retour une série de onze articles intitulée «Israèl ou la dernière chance». La parution de cette série est annoncée en première page de la Presse, le 24 février 1962. Certains des articles qui la composent ont été repris dans Canadian Jewish Digest, volume 3, année 1962.

- «Terre de soleil et de sable»

78:113, 26 février 1962, p. 7.

(sur l'aspect géographique) 
- «Une nation dispersée qui veut être un peuple uni» 78:114, 27 février 1962, p. 5.

- «Le passé non oublié, l'avenir dont on rêve, le présent à découvrir" 78:115, 28 février 1962, p. 5. («au centre, le présent»)

- «La famille, principe fondamental» 78:116, ler mars 1962, p. 5.

- "La politique de la religion ou la religion de la politique» 78:117, 2 mars 1962, p. 5.

- «Une langue, celle de la Bible: l'hébreu» 78:118, 3 mars 1962, p. 35.

- "Une passion collective: l'étude» 78:119, 5 mars 1962, p. 5.

- «Toutes les aspirations: un seul idéal» 78:120, 6 mars 1962, p. 5. (sur Ayel Dayan)

- «Du camp de la mort à la Terre Promise» 78:121, 7 mars 1962, p. 5 . (sur l'immigration)

- «Eichman: dans une cage, un mort vivant» 78:122, 8 mars 1962, p. 5. (sur l'affaire Eichman)

- «Deux mille ans de retard... à reprendre» 78:123, 9 mars 1962, p. 9. (en quittant Israël)

(Cette série fut précédée de l'article suivant: «Noèl à Jérusalem Pour les chrétiens, une trève"), $78: 66,2$ janvier 1962, p. 5 et 6 )

b) Envoyée spéciale de la Presse aux Etats-Unis, Monique Bosco publie cette série de quatre articles

- «En douze ans, la non-violence a réussi» 93:31, 7 février 1977, p. A-9.

- «Le mur de l'argent maintient la ségrégation» 93:32, 8 février 1977, p. A-9.

- «La lutte pour la vie reste à gagner» 93:33, 9 février 1977, p. A-15.

- "L'Amérique est en quête de nouvelles valeurs" 93:34, 10 février 1977, p. A-8.

3- Dans le Devoir

a) Chronique hebdomadaire «Télévision et radio... par Monique Bosco» 
- «Deux créations canadiennes» 53:46, 24 février 1962, p. 13.

- «Par-delà les âges» 53:52, 3 mars 1962, p. 13.

- "Toujours le dimanche» 53:58, 10 mars 1962, p. 12.

- «Bravo à Kanawio!» 53:64, 17 mars 1962, p. 13.

- «Public et 'Premier Plan'» 53:70, 24 mars 1962, p. 13.

- «Présence de l'art, absence des arts» 53:76, 31 mars 1962, p. 12.

- «Deux nouvelles séries» 53:82, 7 avril 1962 , p. 6 .

- «Trois émissions religieuses» 53:88, 14 avril 1962, p. 13.

- «Les mille et une histoires» 53:93, 21 avril 1962, p. 9.

- «Hélas le lundi soir» 53:99, 28 avril 1962, p. 15.

- "Mais où est le théâtre?» 53:105, 5 mai 1962, p. 13.

- «Ni chair, ni poisson, ni rien» 53:111, 12 mai 1962, p. 9.

- «Et vivent les variétés!» 53:117, 19 mai 1962, p. 13.

- "Une heure à tuer...» 53:123, 26 mai 1962, p. 9.

- «Enquête au pays des hommes» 53:129, 2 juin 1962, p. 13.

- «Voici Radio-Canada!» 53:135, 9 juin 1962, p. 13.

- «Du pain et des jeux...» 53:141, 16 juin 1962, p. 10.

- «Dernières éditions» 53:147, 23 juin 1962, p. 13.

- "Famille, que de crimes...» 53:152, 30 juin 1962, p. 13.

- . «Absolvo Te» 53:157, 7 juillet 1962, p. 12.

- "Comment on abrutit les adultes'» 53:163, 14 juillet 1962, p. 12.

- "Machines et machins" 53:169, 21 juillet 1962, p. 13. 
- “"Ayant chanté tout l'été"” 53:175, 28 juillet 1962, p. 13.

- «Au rayon des enfants» 53:181, 4 août 1962, p. 13.

- «'La radio... parfois...'» 53:187, 11 août 1962, p. 15.

- "La radio... encore...» 53:193, 18 août 1962, p. 15.

- «La Tour de Nesle» 53:199, 25 août 1962, p. 10.

- «J'ai deux amours» 53:205, 1er septembre 1962, p. 12.

- «Deux poids, deux mesures...» 53:210, 8 septembre 1962, p. 15.

- "Réchauffé et éclectisme» 53:216, 15 septembre 1962, p. 9.

- “Allons enfants...'» 53:222, 22 septembre 1962, p. 10.

- «Du sérieux... à l'extraordinaire» 53:227, 28 septembre 1962, p. 13.

- «La rentrée des classes» 53:234, 6 octobre 1962, p. 9.

- «Un magnifique téléthéâtre» 53:240, 13 octobre 1962, p. 17.

- "Un retour aux sources" 53:246, 20 octobre 1962, p. 42.

b) Chronique hebdomadaire "La télévision par Monique Bosco»

- "Vive la jeunesse» 53:252, 27 octobre 1962, p. 17.

- «Nos malheurs quotidiens» 53:258, 3 novembre 1962, p. 11.

- «Les trois coups» 53:264, 10 novembre 1962 , p. 11.

- «Loup, où es-tu?» 53:270, 17 novembre 1962, p. 9.

- «Problèmes du réalisme» 53:276, 24 novembre 1962, p. 9.

- «... le rire est difficile» 53:282, ler décembre 1962, p. 14.

- «Art et perfection» 53:288, 8 décembre 1962, p. 14.

- "Les fastes du conformisme» 53:294, 15 décembre 1962, p. 9. 
- «Les limites de l'insolence» 53:300, 22 décembre 1962, p. 13.

- «La vie parisienne» 54:9, 12 janvier 1963, p. 13.

- "'L'éternel mari' trompé» 54:15, 19 janvier 1963, p. 9.

- "Sur des pensers anciens..."» 54:21, 26 janvier 1963, p. 17.

- «Un bijou de perfection» 54:27, 2 février 1963, p. 13.

- «Distractions et éducation» 54:33, 9 février 1963, p. 9.

- «À bout de souffle» 54:39, 16 février 1963, p. 9.

- «Et vive Elisabeth!» 54:45, 23 février 1963, p. 15.

- "Question et inquisition" 54:51, 2 mars 1963, p. 9.

- "'Fin de partie'» 54:57, 9 mars 1963, p. 15.

- «Noblesse oblige!...» 54:63, 16 mars 1963, p. 16.

- «Bonsoir, l'ennui...» 54:69, 23 mars 1963, p. 9.

- «Au jour le jour» 54:75, 30 mars 1963, p. 13.

- «Trop est trop» 54:81, 6 avril 1963, p. 40.

- "La soirée de l'année» $54: 86,13$ avril 1963 , p. 9.

- "Phèdre» 54:98, 27 avril 1963, p. 11.

- «Gala, quand tu nous tiens!» 54:104, 4 mai 1963, p. 13.

- "Ça, c'est du music-hall» 54:110, 11 mai 1963, p. 13.

- «France, Russie, Canada» 54:116, 18 mai 1963, p. 9.

- «Morte saison» 54:128, ler juin 1963, p. 12.

- «Présence de l'art» 54:134, 8 juin 1963, p. 15.

- «Europe, Amérique» $54: 140,15$ juin 1963, p. 12. 
- «Les critiques littéraires de Montréal proposent leurs choix de livres pour Noëli

55:299, 19 décembre 1964, p. 13.

4- $\quad$ Articles divers

- "Contrainte manifeste» Études françaises, Montréal, 16:3-4, octobre 1980, p. 119-131.

- «Lettre ouverte aux critiques littéraires masculins!» le Devoir, 67:113, 17 Mai 1975, p. 24. (On lira là-dessus la réaction de Marie-Andrée Hamel «... mais une colère injuste: la Lettre de Monique Boscon, dans le Devoir, 67:136, 14 juin 1975, p. 18)

- "La moisson de mots de Réjean Ducharme» Europe, Paris, 47:478-479, fév.-mars 1969, p. 72-76.

- "Le Fédéralisme et la Société canadienne-française de Pierre Elliot Trudeau» Études françaises, Montréal, 4:1, fév. 1968, p. 103-104.

- "La fugue d'André Berthiaume» Québec 67, Montréal, 4:10, mai 1967, p. 92-93.

- "La Route d'Altamont de Gabrielle Roy» Québec 66, Montréal, 3:8, octobre 1966, p. 81-82.

- «Aaron de Yves Thériault: séduction de la montagne et dénuement du ghetto" la Presse (Arts et Lettres), 81:78, 3 avril 1965, p. 3.

- «Les éditions de l'Hexagone» le Journal musical canadien, mai 1956, p. 6.

- "Critique des critiques" le Quartier latin, 31:22, 17 déc. 1948, p. 5.

- «Rythmes et mélodies» le Quartier latin, 31:20, 10 déc. 1948, p. 2.

- Aussi quelques articles dans la Revue moderne (août et déc. 1952, mars 1953, août 1954, avril et juin 1955).

\section{H - ENTREVUES}

- LASNIER, Michelle,

- MARCOTTE, Gilles,
"Les quatre romancières de l'année» Châtelaine, 3:6, juin 1962, p. 30, 31, 32, 94, 95, 96, 98, 101, 102. (aussi Suzanne Paradis, Diane Giguère, Marie-Claire Blais) "Les Infusoires de Monique Bosco» la Presse (Arts et Lettres), 81:257, 6 novembre 1965 , p. 5. 
70 VOIX ET IMAGES, VOL. IX, NO 3 (PRINTEMPS 84)

- TREMBLAY, Robert, «La tendre cruauté de Monique Bosco» le Soleil, Québec, 79:49, 22 février 1975, p. D-8.

- BASILE, Jean, «Tout de que j'écris est un plaidoyer pour la liberté") le Devoir, 69:232, 8 octobre 1977, p. 18. 


\section{II - ÉTUDES ET CRITIQUES}

\section{A - SUR LES ROMANS ET RÉCITS}

\section{SUr UN AMOUR MALADROIT}

- S.D.V.

- N.Z.

- ETHIER-BLAIS, Jean

- DUHAMEL, Roger

- MANDEL, Arnold

- MARCOTTE, Gilles

- J.C.

- Anonyme

- Anonyme

- MARCOTTE, Gilles

- MAILHOT, Michèle

- GAY, Paul

- DUHAMEL, Roger
«Un amour maladroit»

le Soir, Bruxelles, 15 juin 1961.

«Un amour maladroit»

France Observateur, Paris, 22 juin 1961.

"Un amour maladroit, de Monique Bosco» Le Devoir, Montréal, 52:158, 8 juillet 1961, p. 11 et 8.

«Un amour maladroit»

la Patrie du Dimanche (Section Magazine), Montréal, 27:30, semaine du 23 juillet 1961, p. 23.

«Les livres - Cendrillon et le baptistère» l'Arche, Paris, no 54, juillet 1961, p. 72-73.

"Un roman de Monique Bosco" la Presse (Arts et Lettres), Montréal, $77: 246,5$ août 1961, p. 18.

«Un amour maladroit»

le Figaro littéraire, Paris, 16:799, 12 août 1961, p. 11.

«Romans - Un amour maladroit» le Nouveau Journal (Cahier des Lettres), Montréal, 1:17, 23 sept. 1961, p. 30. "Un amour maladroit" Bulletin du Cercle Juif, Montréal, 8:68, oct. 1961, p. 2.

«Les livres»

la Presse (Arts et Lettres), Montréal, 78:65, 30 déc. 1961 , p. 3.

"Un beau roman triste"

Châtelaine, Montréal, 2:12, décembre 1961, p. 20.

"Un amour maladroit"

le Droit, Ottawa, 50:153, 30 juin 1962, p. 12.

«Romans et nouvelles - Un amour maladroit»

University of Toronto Quaterly, Toronto, University of Toronto Press, 31:4, July, 1962, p. 550-552. 
- GAY, Paul

- TREMBLAY, Normand

- Anonyme

- RULLIĖRE, Gilbert

- KATTAN, Naïm

- MARCOTTE, Gilles

- Anonyme

Sur LES INFUSOIRES

- MARCOTTE, Gilles

- (Iconographie)
«Un amour maladroit»

Lectures, Montréal, 9:1, septembre 1962, p. 15.

"Un amour maladroit"

Livres et Auteurs canadiens 1961, Montréal, éd. Jumonville, 1962, p. 11 et 12. "Monique Bosco - Un amour maladroit» Bulletin critique du livre français, Paris, août-septembre 1961, fiche no 49565.

"Un amour maladroit" les Fiches bibliographiques, Paris, 1961, fiche no 9013.

«Pour le romancier canadien-français, l'universel c'est l'Afrique»

le Devoir, Montréal, 55:263, 7 novembre 1964, p. 23.

«De Montréal à Venise, l'échec du bonheur"

la Presse (Arts et Lettres), Montréal, 81:275, 27 nov. 1965, p. 4.

«Un amour maladroit»

Mes fiches, Montréal, avril 1966, fiche no 162.

"Les Infusoires de Monique Bosco» la Presse (Arts et Lettres), Montréal, 81:257, 6 nov. 1965, p. 5 (entrevue). «Monique Bosco: Un nouveau roman» Dimanche-Matin, Montréal, 12:41, 7 nov. 1965, p. 61.

- BEAUREGARD, Hermine «Après Marcel Dubé, Monique Bosco» le Petit Journal, Montréal, 40:3, semaine du 14 nov. 1965 , p. 56.

- ÉTHIER-BLAIS, Jean «les Infusoires de Monique Bosco» le Devoir, Montréal, 56:272, 20 nov. 1965, p. 13.

- VALIQUETTE, Bernard «les Infusoires»

Echos-Vedettes, Montréal, 3:44, 20 nov. 1965 , p. 34.

- MARCOTTE, Gilles
«De Montréal à Venise, l'échec du bonheur" la Presse (Arts et Lettres), Montréal, $81: 275,27$ nov. 1965, p. 4. 
- GAY, Paul

- (Iconographie)

- MAJOR, André

- PILON, Jean-Guy

- ROY, Claire

- BERTRAND, André

- LOCKQUELL, Clément

- ETIENNE, Gérard

- Anonyme

- BERTRAND, André

- SAINT-ONGE, Paule

- ALLARD, Marc-Aurèle

- PALLASCIO-MORIN, Ernest «les Infusoires, un roman de Monique Boscon le Droit, Ottawa, 53:274, 27 nov. 1965, p. 7.

le Petit Journal, Montréal 40:5, semaine du 28 nov. 1965, p. 38.

«les Infusoires»

le Petit Journal, Montréal, 40:5, semaine du 28 nov, 1965, p. 34.

«les Infusoires»

Liberté, Montréal, 7:6, nov.-déc. 1965 (no 42), p. 583-4.

«les Infusoires de Monique Bosco - Des larves canadiennes à Venise; trois petits tours et puis s'en vont»" le Nouvelliste, Sherbrooke, 46:31, 4 déc. 1965 , p. 14. «les Infusoires» le Quartier Latin (Le Cahier des Arts et des Lettres), Montréal, 2:10, 9 déc. 1965, p. 7. «les Infusoires》 le Soleil, Québec, 68:294, 11 déc. 1965, p. 6.

«les Infusoires: un livre à lire»

Métro-Express, Montréal, 2:126, 18 déc. 1965, p. 16.

«les Infusoires»

la Patrie, Montréal, 86:50, semaine du 19 déc. 1965 , p. 64.

«les Infusoires»

le Quartier latin (Le Cahier des Arts et des Lettres), Montréal, 2:13, 27 janv. 1966, p. 11. (résumé de l'article du 9 déc. 1965) «Érotisme, révolution et évasion» Châtelaine, Montréal, 7:1, janvier 1966, p. 60.

"Nouveautés - Québec»

l'Alouette, Toronto, 4:2, 2 février 1966, p. 5.

«les Infusoires - Et puis tout est silence...» l'Action, Québec, 59:17,588, 18 fév. 1966, p. 21. 
- MARCOTTE, Gilles

- KATTAN, Naïm

- THERIO, Adrien

- Anonyme

- Fiche J.L.Q.

- ÉTHIER-BLAIS, Jean

- La Rédaction

SUr LA FEMME DE LOTH

- RICHER, Julia

- ÉTHIER-BLAIS, Jean

- Anonyme

- DUCIAUME, J.-M.

- SAINT-ONGE, Paule «les Infusoires»

Québec 66, Paris, Ministère des affaires culturelles du Québec, 3:6, fév. 1966, p. 111-114. «les Infusoires» Bulletin du Cercle Juif, Montréal, 12:112, avril 1966, p. 3. «les Infusoires»

Livres et Auteurs canadiens 1965, Montréal, éd. Jumonville, 1966, p. 63.

«les Infusoires»

Mes fiches, Montréal, juin 1966, fiche no 210.

«les Infusoires»

Vient de paraître, Montréal, 7:2, juin 1971, p. 22 (fiche ouvrage no 23 des Jeunesses Littéraires du Québec).

«Romans - les Infusoires»

University of Toronto Quaterly, Toronto, University of Toronto Press, 35:4, July, 1966, p. 518-519.

"Châtelaine en pantoufles»

Châtelaine, Montréal, 7:10, oct. 1966, p. 2.

«la Femme de Loth, par Monique Bosco» l'Information médicale et paramédicale, Montréal, 23:1, 17 novembre 1970, p. 60. «la Femme de Loth, de Monique Bosco: recréer sa vie» le Devoir, Montréal, 61:259, 7 novembre 1970, p. 11.

"la Femme de Loth"

le Livre canadien (Service de presse), Montréal, Office des Communications sociales, 1:198, 1970 (s.p.).

«la Femme de Loth» Livres et Auteurs québécois 1970, Montréal, éd. Jumonville, 1971, p. 52-53. «Sur des amours condamnées, deux oraisons funèbres...» Châtelaine, Montréal, 12:1, janvier 1971, p. 49. 
- Anonyme

- MARTEL, Réginald

- MARTEL, Réginald

- MARTEL, Réginald

- VACHON, G.-André

- ROBIDOUX, Réjean «la Femme de Loth»

l'Église canadienne, Montréal, 4:1, janvier 1971 , p. 20.

«D'un destin singulier»

la Presse (Arts et Lettres), Montréal, 87:31, 6 février 1971, p. C-3.

«la Femme de Loth" Québec aujourd'hui, Paris, Ministère des Affaires intergouvernementales du Québec, 7:21, mai 1971, p. 172-173.

"la Femme de Loth»

Québec aujourd'hui, Paris, Ministère des

Affaires intergouvernementales du Québec, 7:21, Mai 1971, p. 172-173.

"Une littérature qui se louisianise?»

Études françaises, Montréal, P.U.M., 7:4, novembre 1971 , p. 421 .

«Romans, récits, nouvelles»

University of Toronto Quaterly, Toronto, Un. of Toronto Press, 40:4, Summer, 1971, p. 428.

«In torment beyond the sisterhood» Globe and Mail, Toronto, 29 mars 1975, p. 22.

«Books received»

Montreal Gazette, Montréal, 12 avril 1975. «Monique Bosco's third novel - Ending it all gracefully - French style»

Toronto Star, Toronto, 12 avril 1975. «Love better lived than read but...» Toronto Sun, Toronto, 16 avril 1975. «Book tells Rise, Fall of Family Publishing Firm»

Evening Times Globe, Saint-John (N.B.), 17 avril 1975. «Woman's struggle is subject» Prince George Citizen, Colombie Britannique, 17 avril 1975.

"Rise, Fall of Seccombe House»

Red Deer Advocate, Alberta, 19 avril 1975. "Lot's Wife»

Star Phoenix, Saskatoon, 25 avril 1975. 
- LOUDER, R.S.

- Anonyme

- FERTH, Pierrette

- FERTH, Pierrette

- ISHERWOOD, Coleen

- MELLAMPHY, Ninian

- FERTH, Pierrette

- HAYSOM, Wendy

- VINTCENT, Brian

- SANDLER, Linda
"Protagonist another 'doormat'» Daily Colonist, Victoria (C.B.), 27 avril 1975, p. 6.

«Lot's Wife»

Quill and Quire, 41:4, avril 1975, p. 37. «A look back on life» Vancouver Province, Vancouver, 2 mai 1975.

«History written of Canadian publishers» Woodstock-Ingersoll Sentinel Review, Ontario, 3 mai 1975, p. 6 A.

«Bosco translated: Suspense spices story of a suicide»

Ottawa Citizen, Ontario, 10 mai 1975.

"A valuable addition to enjoyable reading"

London Evening Free Press, Ontario, 10 mai 1975.

«Publishing House Book Tells Story»

Welland Port Colborne Tribune, Ontario, 14 mai 1975.

"Quebec author weaves suspense»

The Spectator, Hamilton (Ontario), 17 mai 1975, p. 4.

"Lot's Wife - One Summer's psalter»

Books in Canada, 4:5, mai 1975; p. 13-14.

«Woman as chattel: a femenist novel from Quebec"

Saturday Night, 90:1, mai 1975, p. 72-75.

- McCORMICK-DELISLE, «Book Review» Sandra

Waterloo Chronicle, 4 juin 1975, p. 23.

- BARCLAY, Pat

«Tears Aplenty in Soggy Saga»

Victoria Times, C.B., 19 juillet 1975.

«Lot's Wife in Quebec»

The Windsor Star, Ontario, 2 aout 1975, p. 44.

- JWG

- FERTH, Pierrette

- WILSON, Betty
"Seeing reality in religion and life»

Prairie Messenger, 10 août 1975, p. 5.

«Book Corner»

Moncton Times, N.B., 30 août 1975.

"Lot's Wife»

The Canadian Author and Bookman, Ontario, 90:4, été 1975, p. 26. 
- Anonyme

- DAVIES, Gillian
«Books by and about women»

The Intelligencer, Belleville Ontario, 8 octobre 1975.

«Lot's Wife»

The Fiddlehead, no 107, automne 1975, p. 137-139.

*L'article de Pierrette Ferth pour la Presse Canadienne est sensiblement le même dans tous les journaux qui l'ont repris.

\section{Sur NEW MEDEA}

- BOURBONNAIS, Josette "L'Année de la Femme, un début!» Dimanche-Matin, Montréal, 22:3, 2 février 1975, p. C-14.

- PELLETIER, Denise «Le drame revécu de Médée et Jason» le Quotidien (du Saguenay-Lac-Saint-Jean), Chicoutimi, 2:108, 8 février 1975, p. C-4.

- MARTEL, Réginald "Les jeux du mythe et du réel» la Presse, Montréal, 91:45, 22 février 1975, p. D-3.

- TREMBLAY, Robert «Donner à l'impossible des dimensions humaines»

le Soleil, Québec, 79:49, 22 février 1975, p. D-8.

- TREMBLAY, Robert "La tendre cruauté de Monique Bosco" le Soleil, Québec, 79:49, 22 février 1975, p. D-8. (Entrevue).

- BASILE, Jean «Un roman bourgeois qui se voudrait mythologique»"

le Devoir, Montréal, 67:50, 1er mars 1975, p. 22.

- LALONDE, Marie «Du courrier - Reproches de poète et de femme»)

le Devoir, Montréal, 67:107, 10 mai 1975, p. 24. (réponse à Jean Basile).

- (Iconographie) «Un nouveau roman de Monique Bosco: New Medean le Saint-Laurent, Rivière-du-Loup, 80:21, 5 mars 1975 , p. 40.

- SAINT-PIERRE, Louise "Médée ou le mariage consumé» Progrès-Écho, Rimouski, 72:8, 12 mars 1975 , p. 49.

- GAY, Paul «Monstrueuse Médée» le Droit, Ottawa, 63:67, 14 juin 1975, p. 20. 
- BLAIS, Marie-Claire

«Le New Medea de Monique Bosco: un livre émouvant...»

le Devoir, Montréal, 67:136, 14 juin 1975, p. 18.

- POULIN, G. et DIONNE, «Romans, récits, nouvelles et contes» $\mathbf{R}$.

University of Toronto Quaterly, Toronto, Un. of Toronto Press, 44:4, Summer, 1975 , p. 321.

- ANDRES, Bernard

«New Medea"

Livres et Auteurs québécois 1975, Québec, P.U.L., 1976, p. 31-32.

- LEBRUN, Paule «New Medea»

Châtelaine, Montréal, 16:9, septembre 1975, p. 24.

- BASILE, Jean "New Medea»

Vient de paraître, Montréal, Edi-Québec, $11: 4$, novembre 1975, p. 43.

Sur CHARLES LEVY, M.D.

- BASILE, Jean

- LEPAGE, Yvan-G.

- ESCOMEL, Gloria

- ESCOMEL, Gloria

- GAUVIN, Lise
«Tout ce que j'écris est un plaidoyer pour la liberté» le Devoir, 69:232, 8 oct. 1977, p. 18. (Entrevue).

"Charles Lévy, m.d.» Livres et Auteurs québécois 1977, Québec, P.U.L., 1978, p. 49-50.

"Monique Bosco ou le miroir brisé» la Nouvelle Barre du jour, Montréal, no 65, avril 1978, p. 90-98.

«Monique Bosco ou la Femme en quête de son double»

Liberté, Montréal, 20:2 (no 116), marsavril 1978, p. 88-95. (le même que précédemment)

«Romans et récits»

University of Toronto Quaterly, Toronto, University of Toronto Press, 47:4, Summer, 1978, p. 341.

SUr PORTRAIT DE ZEUS PEINT PAR MINERVE

- BEAULIEU, Michel «Ah... ces horribles mâles!»

le Livre d'ici, Montréal, 7:45, 11 août 1982 (s.p.). 
- ALONZO, Anne-Marie «Le livre peint»

la Nouvelle Barre du jour, Montréal, no 116, septembre 1982, p. 90-91.

- STANTON, Julie «Portrait de Zeus peint par Minerve» la Gazette des femmes (Voilà la culture), octobre 1982, p. 4.

\section{B - SUR LES POÈMES}

Sur les POÈMES publiés dans Écrits du Canada français

- MARCOTTE, Gilles

- ETHIER-BLAIS, Jean

- GODBOUT, Jacques

- MAILHOT, Michèle

- VACHON, G.-André
«Littérature d'imagination au Canada français» la Presse (Arts et Lettres), Montréal, 79:124, 9 mars 1963, p. 8. "Les Écrits du Canada français, tome XV" le Devoir, Montréal, 54:57, 9 mars 1963, p. 13. «Poésies» Liberté, Montréal, 5:2, mars-avril 1963 (no 26), p. 152-153.

"Châtelaine a lu pour vous: Guerre de religion vingtième siècle» Châtelaine, Montréal, 4:6, juin 1963, p. 44.

"Les Écrits du Canada français» Relations, Montréal, Bellarmin, no 274, octobre 1963, p. 299-300.

«Jéricho ou le lieu commun»

Point de mire, Montréal, 3:7, 27 nov. 1971, p. 40-41.

- GALLAYS, François

- Anonyme

- PILON, Jean-Guy
«Jéricho»

Livres et Auteurs québécois 1971, Montréal, éd. Jumonville, 1972, p. 158.

«Jéricho»

le Livre Canadien (Service de presse), Montréal, Office des Communications sociales, 2:239, 1971 (s.p.). «Les "confidences» de Bosco et Mauffette» le Devoir, Montréal, 63:70, 24 mars 1972, p. 13. 
- DIONNE, René

- MAJOR, Jean-Louis

- MARCOTTE, Gilles

Sur SCHABBAT 70-77

- Anonyme

- PARADIS, Suzanne

- MICHAUD, Ginette

- COLlET, $P$.
"Sur les voies de notre poésie - III" Relations, Montréal, éd. Bellarmin, no 370, avril 1972, p. 122-124.

«Poésie - Jéricho»

University of Toronto Quaterly, Toronto, University of Toronto Press, 41:4, Summer, 1972, p. 350.

«La poésie - Le poète et ses mots»

Etudes françaises, Montréal, P.U.M., 9:1, fév. 1973, p. 85.

«Schabbat 70-77»

Vient de paraître, Montréal, Edi-Québec, 14:4, 1978, p. 19.

«Pour rejoindre le silence»

le Devoir, 70:234, 6 octobre 1979, p. 19.

«Schabbat 70-77»

Livres et Auteurs québécois 1978, Québec, P.U.L., 1979, p. 96-97.

«Les états divisés du monde de Monique

Bosco»

Études canadiennes/Canadian studies, Association française des études canadiennes, Maison des sciences de l'homme d'Aquitaine, Talence, France, no 9, 1980 (publication annuelle).

\section{C - SUR LES TEXTES RADIOPHONIQUES}

- HOULE, René

- Anonyme

- Anonyme

- ESCOMEL, Gloria

- Anonyme
«Plaisir à Zola»

Ici Radio FM, Montréal, no 206, semaine du 17 avril 1976, p. 20.

«Monique Bosco et Venise»

Ici Radio FM, Montréal, no 228, semaine du 20 septembre 1976, p. 9.

«Le cri de la folle enfouie dans l'asile de la mort»

Ici Radio FM, Montréal, no 332, semaine du 18 septembre 1978, s.p.

«Dyne Mousso interprète Monique Bosco» le Devoir, 16 septembre 1978, p. 32. «Monique Bosco lue par Gaétane Laniel» Ici Radio FM, Montréal, no 534, semaine du 2 août 1982, s.p. 


\section{D - SUR LES ARTICLES PUBLIÉS DANS MACLEAN}

- Anonyme

«Les 40 points qui ont fait la saison artistique de Montréal 1964»

le Devoir, Montréal, 55:307, 31 décembre 1964, p. 15.

\section{E - SUR L'ENSEMBLE DE L'OEUVRE}

(On trouvera ici, entre autres, des commentaires de la thèse de doctorat de Monique Bosco, présentée à l'Université de Montréal en 1953, "L'isolement dans le roman canadien-français» (203 p.), non publiée.)

- DUMONT, Fernand et FALARDEAU, Jean-Charles

- DE GRANDPRE, Pierre

- SYLVESTRE, Guy

- ACELF

- Collectif

- MAILHOT, Laurent

- DE GRANDPRÉ, Pierre

Littérature et Société canadiennesfrançaises, Québec, P.U.L., 1964, p. 8, 146-150.

Dix ans de vie littéraire au Canada français

Montréal, Beauchemin, 1966, p. 14, 249.

Panorama des Lettres canadiennesfrançaises

Québec, Ministère des Affaires culturelles, 1967, p. 62.

Esquisses du Canada français

Association canadienne des éducateurs de langue française, Ottawa, 1967, p. 134 (distribué par Fides à Montréal).

Histoire de la littérature française du Québec, t. IV

Montréal, Beauchemin, 1969, p. 16, 58, 94 , 123, 126, 137, 140, 153, 161, 180, 364.

Poèmes 70 - Anthologie des poèmes de l'année au Québec Ottawa, éd. de l'Hexagone/Conseil des Arts du Canada, 1970, p. 11 et 12 (tiré du numéro d'Europe pré-cité).

la Littérature québécoise

Paris, PUF, «Que Sais-je?», no 1579, 1974, p. 90.

- HAMEL, Réginald et al. Dictionnaire pratique des auteurs québécois Montréal, Fidès, 1976, p. 73. 
82 VOIX ET IMAGES, VOL. IX, NO 3 (PRINTEMPS 84)

- MAILHOT, Laurent et NEPVEU, Pierre

- GAUVIN, Lise, MAILHOT, Laurent et al. Montréal, éd. du Boréal Express, 1982, p. 93-94. la Poésie Québécoise - des origines à nos jours, Anthologie Québec/Montréal, Presses de l'Université du Québec/éd. de l'Hexagone, 1980, p. 327 (tiré de Schabbat 70-77). Guide Culturel du Québec 